\title{
Efficiency and Thermography in Cycling during a Graded Exercise Test
}

\author{
Sébastien Duc*, Ahlem Arfaoui, Guillaume Polidori, William Bertucci \\ University of Reims Champagne-Ardenne, France
}

Received: March 03, 2015; Accepted: May 20, 2015; Published: June 13, 2015

*Corresponding author: Sébastien Duc, Research Group in Engineering Sciences ( GRESPI EA 4694), UFR STAPS Reims, Campus Moulin de la Housse, 51687 Reims Cedex 2, France, Tel: +33 (0) 326913 247; Fax: + 33 (0) 326913 250; Email: sebastien.duc@univ-reims.fr

\begin{abstract}
During cycling exercise, at least $75 \%$ of energy expended is wasted as heat or used to maintain homeostasis regard to the consistent value of Gross Efficiency (GE), Net Efficiency (NE) and Delta Efficiency (DE) reported in the literature (18 - 26\%). Therefore, it would be assumed that potential link exists between cycling efficiency and thermoregulation process. Skin temperature of two lower limb muscle's group (quadriceps and calves) detected by infrared camera and cycling efficiency parameters determined by gas exchanges analysis were measured during a graded exercise test until exhaustion in seven trained young cyclists.
\end{abstract}

Unlike to GE (median (interquartile range) 19.8 (2.0)\%) and NE (22.4 (3.3)\%), DE (26.1 (2.7)\%) was significantly inverse correlated $(\mathrm{r}=-0.821 ; p=0.034)$ with relative $(-8.6(1.9) \%)$ changes in skin temperature over VL when power output increases from 100 to 300 $\mathrm{W}$. These results suggested that even if GE and NE were unrelated to the thermoregulation effectiveness, DE seems to be higher in cyclists who showed the highest variation of skin temperature over the quadriceps muscle. test

Keywords: Thermography; Delta efficiency; Cycling; Incremental

\section{Introduction}

Cycling efficiency can be assessed through the determination of the GE, i.e. the ratio of the total amount of the effective mechanical work generated by the muscles to the total energy expended to do that work [1]; the NE with subtracting the energy expended at rest to the total energy expended which implicitly assumes that during exercise, energy expenditure is constant and independent of mechanical work [2]; or the DE which corresponds to the change in effective mechanical work performed divided by the change in total energy expended [1]. DE can also be computed by the reciprocal of the slope of the linear relationship between energy expenditure and mechanical work [3]. Even though it is less reproducible than GE, DE has been used to estimate the muscular efficiency since the influence of the maintenance of homeostasis on the total energy expenditure is removed [4].

It is well known that muscle exercise like pedalling involves an increase in internal temperature due to the heat accumulation which represents the difference between the energy consumption and the mechanical energy. With regard to the consistent values of GE, NE, DE reported by the literature [1-4], i.e between $\sim 18$ to $26 \%$, respectively it can be assumed that at least $70 \%$ of all the energy expended from ATP hydrolysis is wasted as heat production [2]. To maintain quasi-constant the internal temperature, the heat flow can be regulated by increasing skin blood perfusion both in the superficial and deep skin layers. However during exercise, the most effective mechanisms are the heat conduction via blood circulation and the cooling by sweat evaporation at the body surface byconvection [5]. Therefore, the skin layer plays an important role in the complex thermoregulatory process as it represents the exchange barrier between the inside of the body and the surrounding environment.

Infrared thermography can be defined as a safe and noninvasive technique for measuring heat radiation on the skin, which permits to estimate temperature from volumes surfaces [6]. Thermal imaging cameras detect radiation in the infrared range of the electromagnetic spectrum and produce images of that radiation, called thermograms. This method provides realtime, instantaneous visual images and video with measurements of surface temperatures over a large distance. Moreover, it permits the quantitative evaluation of specific cutaneous whole body thermal adaptations which occur during and after graded exercise activity [7]. For these practical reasons, several researchers have turned to this method as it can easily provide information on heat production and dissipation during local muscular exercise $[8,9]$ or during whole body exercise [7,10-13]. Nevertheless, the use of infrared thermography presents some methodological aspects [14]. For example, when perspiration is important, infrared radiation could be decreased due to the skin cooling. Therefore, infrared measures could be changed when emissivity of the skin is altered [15]. Subcutaneous fat tissue can also influence infrared measured due their insulating capacity in heat transfer [16]. A recent study by Quesada et al. [17] reported lower change in skin temperature over quadriceps muscle during an incremental cycle exercise in subjects with larger thigh skin fold.

By thermography, several studies observed a sudden fall of skin temperature immediately after the beginning of a dynamic 
exercise $[7-9,11,13]$. Although the mechanism is not yet fully understood, some authors have suggested that initial reduction in skin surface is rather due to the vasoconstriction of cutaneous vessels for increase the muscular blood flow of the working mucles than thermoregulation process $[18,19]$. However, as exercise progresses, the continuous decrease of skin temperature is more related to the cooling due to the sweat evaporation. The human body evacuates the heat accumulation with its environment much more than the rest and the skin surface is rapidly cooled. Eighty percent of the blood flow is estimated to be devoted to the process of thermoregulation [9].

Johnson et al. [20] tried to determine whether skin is relatively vasoconstricted during upright exercise in the heat and to determine the roles of upright posture and exercise on the relationship of skin blood flow to internal temperature. They conclude that increasing blood flow was a result of competition between reflex vasoconstriction with muscular work. They suggested that skin is vasoconstricted during heat stress with exercise or upright posture and that the greatest effect occurs when these stresses are combined. Indeed, Torii et al. [13] focused on the skin temperature distribution of cycling men during initial muscular work. Eleven healthy men were selected to take part in a bicycle exercise at workloads of $50-150 \mathrm{~W}$ in a climatic chamber at ambient temperatures of $10-40^{\circ} \mathrm{C}$ (relative humidity 45 - 55\%). They showed that increased work intensities reduced skin temperature. These results suggest that the fall in skin temperature during initial exercise was not due to increased evaporative cooling but to vasoconstriction. The vasoconstriction is due to the muscular blood recruitment, prolonging exercise increases metabolic heat production and invokes thermal regulatory processes.

One recent study observed a strong relationship between the decrease of skin temperature and the increase of heart rate in master's cyclists during an incremental exercise test [11]. A continuous circulation of air flow is provided to the skin by the leg's movement while pedaling. The heat convection can explain also the high decrease of the skin temperature. However, it seems difficult to distinguish if this decrease is due to vasoconstriction only or to the combination between vasoconstriction and convection [18].

It could be possible that differences in cycling efficiency are related to the subject's ability for heat elimination. Regards to the theoretical framework of thermoregulation process, we hypothesized that the thermoregulation efficiency can be reflected by changes in the skin temperature. In this line, cyclists with higher efficiency would showed the higher fall of skin temperature over the muscle while exercise intensity increases. At our knowledge, not many studies have investigated the potential relationships between efficiency in cycling and thermoregulation assessed by infrared thermography, but one study have shown that infrared thermography based results related to thermoregulation were obtained and compared in the well-trained and the ordinary cyclists [14].

The other had shown that the trained subjects (professional footballers) have a better vasoconstriction capability and a better capacity to dissipate through the skin layers the metabolic heat produced than untrained subjects [21].

The aim of this study was to examine the possible relationships between whole body (GE, NE) and muscular efficiency DE and specific cutaneous whole body thermal adpatations assessed by changes in skin muscle thermograms during a graded cycling exercise.

\section{Methods}

\section{Participants}

Seven trained young male competitive cyclists volunteered to participate in this study (mean \pm SD: age: $16.1 \pm 0.9$ years; stature: $1.80 \pm 0.06 \mathrm{~m}$; body mass: $63.9 \pm 4.9 \mathrm{~kg}$; maximal oxygen uptake $\left(\mathrm{VO}_{2 \max }\right): 61.7 \pm 5.9 \mathrm{~mL} \cdot \mathrm{kg}^{-1} \cdot \mathrm{min}^{-1}$; peak power output $\left(\mathrm{P}_{\max }\right): 325 \pm$ $31 \mathrm{~W})$. The subjects were asked to avoid smoking, alcohol, coffee within three hours before the exercise [22] and to refrain from intense exercise for at least 24 hours before their participation in the study. We made sure that participants did not drink just before and during the test, because taking drinks affects the process of thermoregulation [23,24]. The subjects must not apply creams, gels and especially the menthol that has an effect on thermoregulation during exercise $[25,26]$. All of subjects were free from known cardiovascular and respiratory diseases at the time of the study.

\section{Experimental design}

Prior to testing and after having received full explanation about the nature and purpose of the study, the subjects and parents for minors gave written informed consent. The study was conducted in accordance with the ethical guidelines of the Declaration of Helsinki [27]. Institutional Review Board for this study was obtained from the ethical committee of University of Reims Champagne Ardenne.

All the tests were performed in laboratory during the off phase of the cycling competitive season (October-November). The laboratory conditions were held constant (ambient temperature $20 \pm 0.5{ }^{\circ} \mathrm{C}$, relative humidity $55 \pm 5 \%$ ) throughout the tests. Before the testing, 30 min were needed to balance the subject's body temperature with the lab environment. Each subject have to wear any clothes on the thighs and legs for performing infrared thermographic measures.

\section{Protocol}

Each subject performed a graded cycling exercise test (GXT) to measure maximal oxygen uptake $\left(\mathrm{VO}_{2 \max }\right)$, maximal heart rate $\left(\mathrm{HR}_{\max }\right)$, peak power output $\left(\mathrm{PO}_{\max }\right)$ and cycling efficiency (GE, NE, $\mathrm{DE})$. The GXT started at $100 \mathrm{~W}$ and the work rate was increased by steps of $40 \mathrm{~W}$ every $4 \mathrm{~min}$ until exhaustion. Throughout the GXT, the subjects could choose their preferred pedalling cadence but were told not vary it more than \pm 3 rev. $\mathrm{min}^{-1}$. They have to remain seated during the test. They were cooled using an electric fan placed in the front.

$\mathrm{P} 0_{\max }$ was calculated according to following equation [28] :

$$
P O_{\max }=P O_{\text {last }}+\left(\frac{t \times 40}{240}\right)
$$


Where $\mathrm{PO}_{\text {last }}$ is the last completed work rate $(\mathrm{W})$ and $\mathrm{t}$ is the time for the incomplete work rate (s).

\section{Techniques and materials}

Before performing the GXT, body mass and stature of the subjects were measured with an electronic scale and stadiometer. The subjects performed the GXT with their own racing bike which was mounted on a stationary cyclotrainer (Fortius, Tacx, Assenar, Netherlands) [Figure 1]. It has been shown that the Fortius cyclotrainer provides a valid and reproducible power output $[29,30]$. The same rear wheel (DT Swiss AG, Biel, Switzerland) was fitted to each participant's bike, inflated to $120 \mathrm{psi}$, and the front fork of the bicycle was attached to the accompanying steering apparatus for ensure the stability and the security of the bicycle. Each participant used his own cycling shoes and clipless pedals to perform the GXT. Power output (PO) and pedalling cadence were recorded continuously during the GXT every second.

Pulmonary ventilation $\left(\mathrm{V}_{\mathrm{E}}\right)$, oxyen uptake $\left(\mathrm{VO}_{2}\right)$, carbon dioxide production $\left(\mathrm{VCO}_{2}\right)$ and Respiratory Exchange Ratio (RER) which was computed by ratio between the amount of $\mathrm{CO}_{2}$ produced and $\mathrm{O}_{2}$ consumed, were measured continuously throughout the GXT via breath-by-breath telemetric open circuit spirometry (Oxycon Mobile, Jaeger, Wurzburg, Germany). The gas analyser was calibrated before each test with gases of known concentration $\left(15.53 \% \mathrm{O}_{2}\right.$ and $5.25 \% \quad \mathrm{CO}_{2}$, Jaeger). Volume and flow were calibrated according to the manufacturer's specifications. The participants wore a facemask and breathed through a low resistance impeller turbine. Heart rate was measured continuously throughout the test using short range radio-telemetry (Polar S800, Polar Electro Oy, Kempele, Finland). Achievement of $\mathrm{VO}_{2 \max }$ was taken as the highest $30 \mathrm{~s}$ value attained before volitional exhaustion.

Gross Efficiency (GE) and Net Efficiency (NE) were subsequently calculated from measures of $\mathrm{VO}_{2}\left(\mathrm{~mL} \cdot \mathrm{min}^{-1}\right) \mathrm{VCO}_{2}$ $\left(\mathrm{mL} \cdot \mathrm{min}^{-1}\right)$, and PO (W) averaged between the $3^{\text {th }}$ and $4^{\text {th }}$ min of the workload step correspond to $220 \mathrm{~W}$. We assumed that once RER rose consistently above 1.00 or an entire workload, the measures of energy expenditure were no longer valid due to the

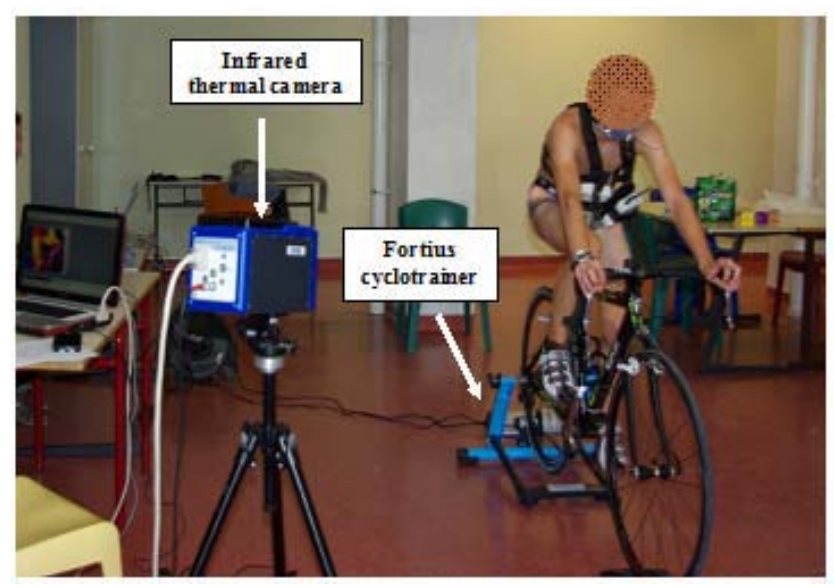

Figure 1: The experimental setup of the GXT test. contribution of unmeasured anaerobic work.

Energy Expenditure (EE) was calculated using the formula of Brouwer [31]:

$$
E E(J)=\left[\left(3.869 \times V O_{2}\right) \pm\left(1.195 \times V \mathrm{CO}_{2}\right)\right] \times 4.186
$$

GE and NE were determined using the following equations:

$$
\begin{aligned}
& G E(\%)=\frac{P O}{E E} \times 100 \\
& N E(\%)=\frac{P O}{E E-E E_{\text {rest }}} \times 100
\end{aligned}
$$

EE at rest was measured in sitting position during 5 minutes for computed NE.

DE (\%) was calculated as the reciprocal of the linear trend line joining the points on an EE versus work rate plot between 100 and $300 \mathrm{~W}$.

Skin temperatures of the right calve (GM: gastrocnemius medialis) and left quadriceps (VL: vastus lateralis) muscles were measured using an infrared thermal camera (FLIR SC1000; 256 $\times 256$ focal plane array; $200 \mathrm{~Hz}$ frame rate; quantum efficiency > $70 \%$ ). The acquisition frequency of the camera was $50 \mathrm{~Hz}$. The minimum detectable temperature difference was less than 0.1 ${ }^{\circ} \mathrm{C}$ in the study temperature range. This type of camera allowed acquisitions even when the study area was moved. The infrared camera was positioned on a tripod at a height of $0.5 \mathrm{~m}$, and at a distance of $1.5 \mathrm{~m}$ at the right side of the bicycle. Infrared images were taken continuously $5 \mathrm{~min}$ before and throughout the GXT. To define and individuate a region of interest, we have used thermally neutral markers placed surrounding the GM and VL muscles as similarly used in one past study. But, unlike most studies in literature that use large areas of the human body to view maps of temperature $[10,21]$ we have chosen to interest only a small area, namely right calve (GM) and left quadriceps (VL), the role of which is crucial in cycling [11]. The first thermal image was used as a reference for the other ones and considered to the rest value. Thermal images were taken every five seconds. As the movement of the legs is cyclic, the position of the calves and the hamstrings is reproducible from one cycle to another. In such a way, it is thus easy to identify the muscles area that will serve as study area from one cycle to another, and to avoid possible artefacts. Changes in skin temperatures $(\Delta \mathrm{T})$ of each muscle were computed against the initial reference (rest) and expressed in percentage.

\section{Statistical analysis}

All statistics were performed using the software Statistica 7 (Statsoft, USA). As the normality of data was not guaranteed, no-parametric tests were used (Spearman correlation analysis) to investigate the relation between gross efficiency and absolute or relative changes in muscle skin temperature $(\Delta \mathrm{T})$. Friedman rank-repeated measures test, Wilcoxon rank-sum test and Mann-Whitney U were also used to study the effect of workload 
on the skin temperature (paired samples) and to determine the differences between the quadriceps and calf muscles (two independent samples). The level of significance was set at $p<$ 0.05 for all tests. Descriptive data are presented as median and Interquartile Range (IQR) except where describes otherwise.

\section{Results}

The results obtained during the GXT are presented in Table 1. The validity of the estimation of DE can be found by examining the accuracy of the trend line passing through the points on an energy expended versus work rate plot. The mean coefficient of determination $\left(\mathrm{r}^{2}\right)$ for the linear trend lines linking the points on the graphs was 0.995. Mean and Standard Deviation of power output during successive workload stages of the GXT was $99 \pm$ $6 \mathrm{~W}, 139 \pm 1 \mathrm{~W}, 179 \pm 1 \mathrm{~W}, 219 \pm 1 \mathrm{~W}, 259 \pm 1 \mathrm{~W}, 299 \pm 1 \mathrm{~W}$ and $345 \pm 5 \mathrm{~W}$, respectively. Mean ( \pm SD) preferred pedalling cadence during the GXT for all subjects was $94 \pm 8$ rev. $\mathrm{min}^{-1}$. The mean $( \pm$ SD) coefficient of variation of pedalling cadence between the first work stage and the last work stage in which RER was below to 1.00 for all subjects was $3.9 \pm 0.7 \%$.

Figure 2 shows an example of thermographic images obtained during the GXT. Skin temperature of VL and GM muscle decreased consistently whilst heart rate increased continuously during GXT (Figure 3). After the GXT, the skin temperature increased suddenly while the heart rate decreased quickly due to the passive recovery of the subject. The decreases (mean \pm SD) of the skin temperature of VL and GM muscles at the end of the GXT for all subjects were $-2.6 \pm 0.6{ }^{\circ} \mathrm{C}(-8.5 \pm 2.0 \%)$ and $-2.7 \pm 0.6{ }^{\circ} \mathrm{C}$ $(-8.7 \pm 1.9 \%)$, respectively. The skin temperature over the muscle at rest and for each workload of GXT are presented in Table 2. The highest completed workload ( $340 \mathrm{~W})$ was excluded of the analysis because only four subjects have started this workload stage and only two have completed it. There was a significant effect of power output on skin temperature of VL and GM muscles $(P<0.0001)$. The skin temperature was not significant different between the two muscles investigated $(P=0.438)$.

There were no significant relationships between GE or $\mathrm{NE}$ and $\Delta \mathrm{T}$ of $\mathrm{VL}(P=0.906$ and $P=0.662$, respectively $)$ or GM ( $\mathrm{P}=0.713$ and $P=0.906$, respectively). In contrast, $\mathrm{DE}$ was significantly correlated with changes of the skin temperature of VL expressed in \% to rest values $(\mathrm{r}=-0.821 ; P=0.034)$. There was also a tendency for $\mathrm{DE}$ to be inverse correlated with changes of skin temperature for GM $(\mathrm{r}=-075 ; P=0.07)$ (Figure 4).

Table 1: Subject's Characteristics, Physiological Data and Cycling Efficiency Parameters Obtained during the Graded Exercise Test. Data are presented as median and Interquartile Range (IQR).

\begin{tabular}{|c|c|}
\hline Measures & Group $(N=7)$ \\
\hline $\mathrm{PO}_{\max }(\mathrm{W})$ & $334(46)$ \\
\hline $\mathrm{HR}_{\max }\left(\right.$ beat.min $\left.{ }^{-1}\right)$ & 205 (16) \\
\hline$\dot{V} 02_{\max }\left(\mathrm{mL} \cdot \mathrm{kg}^{-1} \cdot \mathrm{min}^{-1}\right)$ & $63.6(7.9)$ \\
\hline GE (\%) - 140W & $19.8(2.0)$ \\
\hline NE (\%) - 140W & $22.4(3.3)$ \\
\hline DE (\%) & $26.1(2.7)$ \\
\hline
\end{tabular}

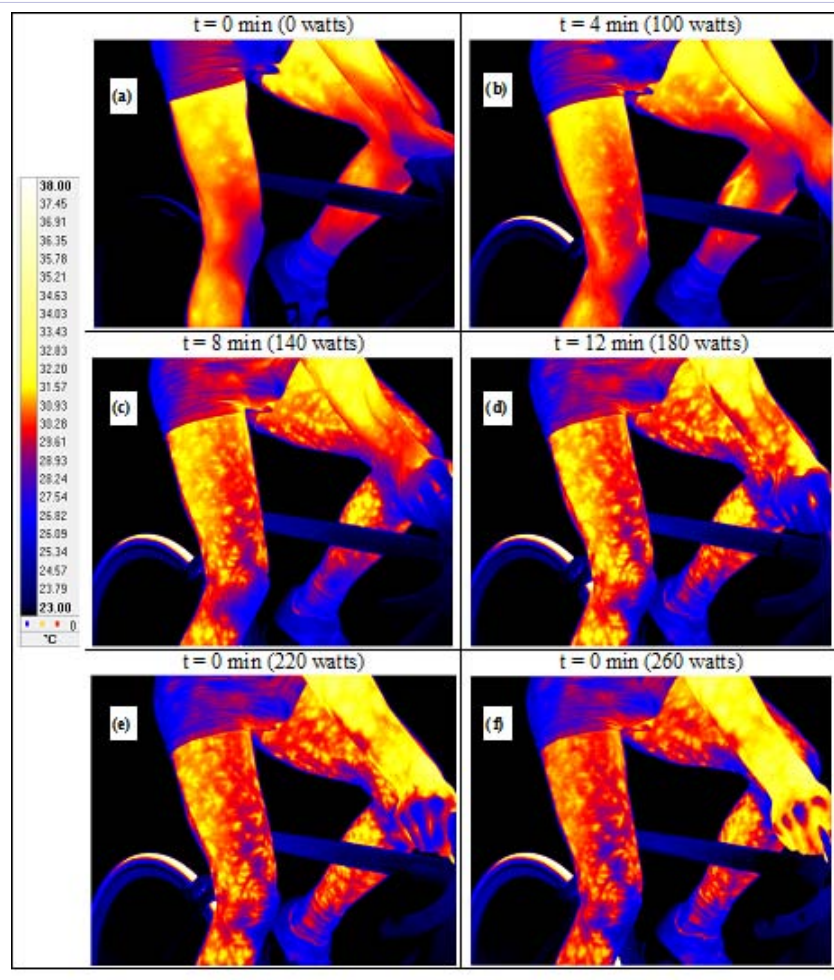

Figure 2: Example of thermal photography during the test.

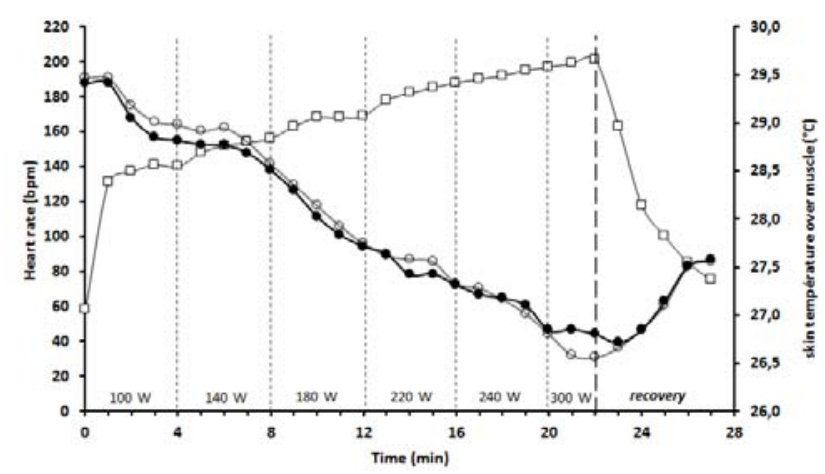

Figure 3: Heart rate $(\square)$ and skin temperature over quadriceps (o) and calve $(\bullet)$ before (rest: $0 \mathrm{~W}$ ), during, and after (recovery) the graded exercise test for one typical subject.

Significant correlations were found between the changes of skin temperature of VL and $\mathrm{HR}(\mathrm{r}=-0.80 ; P<0.001)$ or $_{2}(\mathrm{r}=-$ $0.72 ; P<0.001$ ) (Figure 5). Similar significant strong correlations were also found for the $\Delta \mathrm{T}$ of GM and HR $(\mathrm{r}=-0.77 ; P<0.001)$ or $\mathrm{VO}_{2}(\mathrm{r}=-0.64 ; P=0.001)$.

\section{Discussion}

The main finding of the present study was that, unlike to GE and NE, DE was correlated with the decrease of the skin temperature of VL muscle during an incremental graded exercise test in trained cyclists. We also found strong relationships between the changes of heart rate and oxygen uptake and the variation of skin temperature of VL and GM muscles. 
Table 2: Skin Temperature of Quadriceps $\left(\mathrm{T}_{\mathrm{Q}}\right)$ and Calve Muscles $\left(\mathrm{T}_{\mathrm{C}}\right)$ during Rest ( $0 \mathrm{~W}$ ) and each Workload (PO) of the Graded Exercise Test. Variation of Skin Temperature $(\Delta \mathrm{T})$ to Rest Value are expressed in \%. Data are presented as median and Interquartile Range (IQR).

\begin{tabular}{|c|c|c|c|c|}
\hline $\begin{array}{c}\mathbf{P O} \\
\mathbf{( W )}\end{array}$ & $\begin{array}{c}\mathbf{T}_{\mathrm{Q}} \\
\left.\mathbf{(}^{\circ} \mathbf{C}\right)\end{array}$ & $\begin{array}{c}\mathbf{T}_{\mathrm{C}} \\
\left.\mathbf{(}^{\circ} \mathbf{C}\right)\end{array}$ & $\begin{array}{c}\mathbf{\Delta} \mathbf{T}_{\mathrm{Q}} \\
\mathbf{( \% )}\end{array}$ & $\begin{array}{c}\mathbf{\Delta} \mathbf{T}_{\mathrm{C}} \\
\mathbf{( \% )}\end{array}$ \\
\hline $\mathbf{R e s t}$ & $31.4(1.1)$ & $31.7(1.5)$ & - & - \\
\hline $\mathbf{1 0 0}$ & $30.9(0.9)$ & $30.9(1.2)$ & $-1.3(0.4)$ & $-1.6(1.0)$ \\
\hline $\mathbf{1 4 0}$ & $30.5(1.6)$ & $30.5(1.4)$ & $-3.0(2.0)$ & $-2.5(1.1)$ \\
\hline $\mathbf{1 8 0}$ & $29.4(1.2)^{\mathrm{ab}}$ & $29.6(1.8)^{\mathrm{ab}}$ & $-5.1(1.4)^{\mathrm{ab}}$ & $-4.2(2.0)^{\mathrm{ab}}$ \\
\hline $\mathbf{2 2 0}$ & $29.3(1.2)^{\mathrm{ab}}$ & $29.5(1.8)^{\mathrm{ab}}$ & $-6.2(1.5)^{\mathrm{ab}}$ & $-4.7(1.7)^{\mathrm{ab}}$ \\
\hline $\mathbf{2 6 0}$ & $29.2(1.5)^{\mathrm{abcd}}$ & $29.1(1.9)^{\mathrm{abcd}}$ & $-6.9(1.9)^{\mathrm{abcd}}$ & $-5.9(2.1)^{\mathrm{abcd}}$ \\
\hline $\mathbf{3 0 0}$ & $28.8(1.7)^{\mathrm{abcde}}$ & $29.1(2.3)^{\mathrm{abcde}}$ & $-7.9(2.8)^{\mathrm{abcde}}$ & $-6.5(3.9)^{\mathrm{abcde}}$ \\
\hline
\end{tabular}

Notes: Significant differences compared to $100 \mathrm{~W}^{(\mathrm{a})}, 140 \mathrm{~W}^{(\mathrm{b})}, 180 \mathrm{~W}^{(\mathrm{c})}$, $220 \mathrm{~W}^{(\mathrm{d})}$ and $260 \mathrm{~W}^{(\mathrm{e})}$ value.

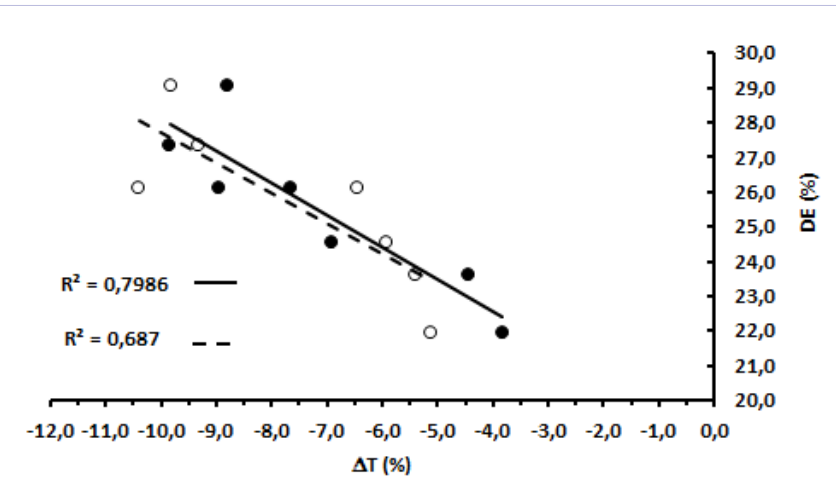

Figure 4: Relationships between delta efficiency (DE) and changes in skin temperatures $(\Delta \mathrm{T})$ expressed in percentage from the rest values during the GXT. (Quadriceps:•, continuous regression line. Calve: o, dashed regression line).

To our knowledge, this is the first investigation about the potential relationship between cycling efficiency and thermoregulation effectiveness assessed by thermography measurement. The strong negative relationship $(r>-0.70)$ between $\mathrm{DE}$ and changes of skin temperature gradient $(\Delta \mathrm{T})$ was only significant for VL muscle. Three cyclists showed the greatest DE ( $>25 \%)$ and $\Delta \mathrm{T}$ at the end of the GXT ( $>-2.25$ ${ }^{\circ} \mathrm{C}$ and $>-7.5 \%$, respectively). In contrast, lowest DEs $(<25 \%)$ were observed in the four cyclists who were characterised by small absolute $\left(<-2.25^{\circ} \mathrm{C}\right)$ and relative $(<-7.5 \%)$ decreases of skin muscle temperature. Larger decrease of skin temperature created higher thermal gradient between the core and the skin, which increase theoretically the heat storage capacity and reducing thermoregulatory strain. This physiological advantage would be related to i) the lower heat production, ii) the higher heat elimination, or iii) the combination of the two mechanisms.

With referring to the reciprocal slope of work rate - metabolic rate relationship, we found a DE of $25.5 \pm 2.4 \%$ (mean \pm SD). This mean value is similar to those reported by previous studies, i.e. between $24-26 \%[1,4,32,33]$. Compared to GE, it is generally accepted that DE is likely to be less affected by potential changes in the baseline energy cost caused by the work rate and knowledge about resting metabolic rate is not required, unlike to NE. According to Ettema et al. [2], only GE is to be considered a "true" efficiency of the whole body. However, DE can be viewed as an estimate of muscular efficiency [4]. In this case, it would be assumed that DE is independent of the work rate increment only if the metabolic cost - work rate relationship is linear. Besides, as it has been underlined in a previous critical review by Ettema et al. [2] a linear relationship between work rate and metabolic rate does not imply that DE provides a valid measure of muscular efficiency, and neither does the finding that the efficiency value obtained are realistic (i.e. between 24 and 26\%). Moreover, Moseley et al. [3] reported higher coefficient of variation of intra-subjects DE measurements (5 - 10\%) compared to GE (3.2 - 6.4\%). Therefore, regards to all these limits and criticisms, the significant relationship observed in the present study between DE and skin temperature gradient must be interpreted with high caution. That is why we cannot affirm that cycling efficiency is related to thermoregulation process assessed by thermography.

Recently, Quesada et al. [17] observed a significant inverse relationship between changes in skin temperature and changes in overall neuromuscular activation for VL during an incremental cycle exercise in ten physically active subjects but with low fitness level (peak power output: $252 \pm 36 \mathrm{~W}$ vs $325 \pm 31 \mathrm{~W}$ for the participants of the present study). According to their results, they concluded that effectiveness adaptive response of
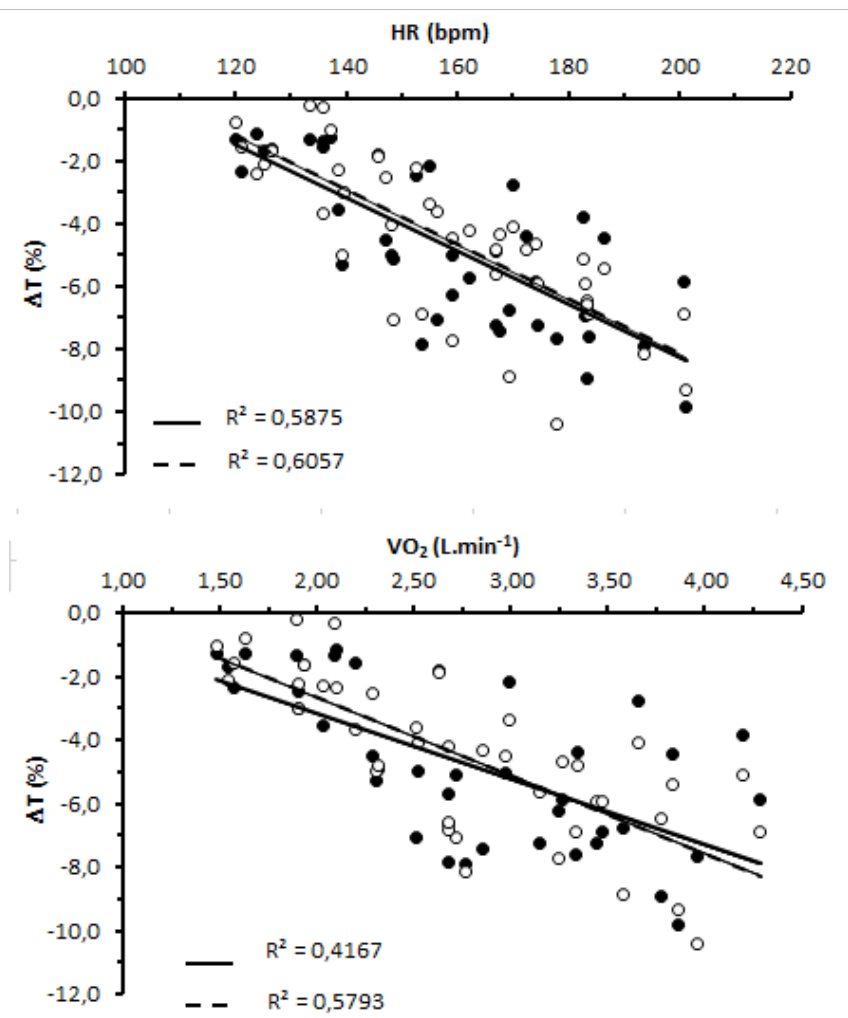

Figure 5: Relationships between Heart Rate (HR) / oxygen consumption (VO2) and changes in skin temperatures $(\Delta \mathrm{T})$ expressed in $\%$ from the rest values during the GXT. (Quadriceps:•, continuous regression line ; Calve: 0 , dashed regression line). 
thermoregulation system showed by changes in skin temperature are related to muscular activation of VL. The inverse relationship between DE and change of skin temperature gradient observed during a graded exercise test in the present study seems to be in accordance with these results. Even if at our knowledge none study have showed that DE and overall muscular activation are linked in cycling, we can suppose that more DE is important, more the muscular efficiency is higher, and therefore less neuromuscular activation is needed. Nevertheless, it is important to emphasise that the research group of Quesada et al. [17] have performed thermography measurement in a standing upright resting position, before, immediately after and $10 \mathrm{~min}$ after finishing the cycling test but not during the incremental exercise. The authors considered the comparison of the skin temperature before and after the incremental test reflects the changes of skin temperature. Therefore, the reported increased of skin temperature in VL would have been confounded by the sudden increase of skin temperature observed just after the end of exercise, as it has been observed by Vainer [14] and in this study (Figure 3). Future studies should be performed to measure simultaneously the neuromuscular activation and infrared thermography during incremental or exhaustive exercise in order to confirm or not the potential link between the effectiveness of thermoregulation response and muscle recruitment.

Concerning the thermographic analysis, the infrared images (Figure 2) were taken during the different phases of the exercise: at rest and during each stage of the incremental progressive exercise. One may note that the skin temperature of right calf and left quadriceps decreases during the exercise. This decrease was no longer homogeneous. Indeed, the relatively homogeneous temperature distribution at the beginning (Figure 2(a)) is replaced by a temperature pattern with distinctly warmer and cooler areas (Figure 2(b)-(f)). This observation is in accordance with the works of Merla et al. [7] who studied cutaneous temperature variations in well-trained runners during graded treadmill exercise until reaching their individual maximal heart rate. They concluded that when performing graded exercise, the subject should favour vasoconstriction of the cutaneous vessels increasing the blood flow to muscles it is the reason why the skin temperature decreases. Moreover, one can observe that the skin temperature distribution presents hyper-thermal spots due to the presence of perforator vessels which reach the surface of skin (Figure 2(f)). The apparition of more elevated temperature spots (the number and arrangement of which seem to vary from one subject to another) has already been observed and discussed by Vainer [14]. It should be noted that, as the cyclic movement of pedalling, the position of the calf and the quadriceps is reproducible from one cycle to another. But it is useful to use a markers recognition algorithm for have more precision.

As it is shown in Figure 1, the skin temperature of the two muscles (VL and GM) fell as soon as the GXT began while HR increased continuously. One recent study observed the same phenomenon in master's cyclists while they performed an incremental exercise test [11]. Their results suggest that as the exercise intensity progresses, the blood flow increase, the more heat is removed and consequently the more the skin temperature decreases. Increased work intensity improve the metabolic heat production and invokes thermal regulatory processes. This results in a continuous fall of the skin temperature with the increase of exercise intensity.

Figure 2 shows also a strong relationship between the decrease of skin temperature and the oxygen consumption during the GXT. It is well known that the increase of work intensity during a muscular exercise enhance higher heat production which represents the difference between the energy expenditure and the mechanical energy ( $\sim 80 \%$ in this study based on the value of GE). The rate of energy expenditure is calculated from the measures of oxygen consumption and carbon dioxide production. Therefore, it is easily seen that the higher the oxygen consumption increases during the muscular effort, the more heat is removed and consequently the temperature of the skin decreases. Predictive equations show when the HR increases to $10 \mathrm{bpm}$, skin temperatures of quadriceps or calf muscles decrease from $0.29^{\circ} \mathrm{C}$ and $0.32^{\circ} \mathrm{C}$, respectively, which represent a relative reduction of $\sim 1 \%$ of rest values. When the $\mathrm{VO}_{2}$ increases

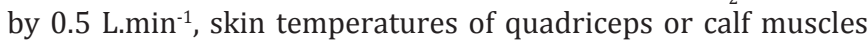
decrease from $0.32^{\circ} \mathrm{C}$ and $0.39^{\circ} \mathrm{C}$, respectively which represent a relative reduction of $\sim 1$ and $\sim 1.3 \%$ of rest values.

Two main limits must be considered and might confound these results. Firstly, we have calculated the cycling efficiency at the same workload stage but not necessarily at the identical pedalling cadence since each participant could select it throughout the GXT. It is well-known that pedalling cadence affects cycling efficiency [2]. Most studies report a clear negative influence of cadence on gross efficiency $[1,32,34,35]$. However, the effect of cadence on DE remains unclear as Nickleberry et al. [36] reported a decrease in DE (27 to $21 \%$ ) as a function of cadence whilst Sidossis et al. [35] observed an increase in DE (20.6 to 23.8\%). However Marsh et al. [33] found no significant difference of DE in experienced cyclists when pedalling cadence varied from 50 rev. $\mathrm{min}^{-1}$ to 110 rev. $\mathrm{min}^{-1}$ and power output from $100-200 \mathrm{~W}$. If one assumes that resting metabolic rate and the cost of limb movements during pedalling is physically independent of work rate, DE would be unaffected by cadence as it is a measure of the increasing cost directly linked to increasing muscle work. In our study, pedalling cadence chosen by the subjects during the GXT, varied between 83 to 104 rev. $\mathrm{min}^{-1}$, with a coefficient of variation of $3.9 \%$, across the workload stage. Therefore, regards to the small intra- and inter-subject variations of the cadence $(\sim 3-4$ and $\sim 20$ rev.min 1 , respectively) we can assign the differences in the parameters of cycling efficiency to the subject's physiological characteristics rather than to the variability of the pedalling cadence.

Secondly, the time of power output steps during GXT (4 min) to measure gas data for the calculation of cycling efficiency (GE, $\mathrm{NE}$ and DE) is much lower to those of recommended by Hopker et al. [28] i.e. 8 min. Nevertheless, De Koning et al. [37] showed that GE values obtained during a maximal incremental test were similar with using 3 or 6 min steps. Moreover, they suggested that GE can be determined robustly so long as steady state exercise is performed and RER 1.00. In this study, to respect these recommendations, we have computed the cycling efficiency 
parameters with using the data collected during the last min of each workload stage and until the RER exceeded 1.00.

\section{Conclusion}

This study showed that the changes of skin temperature of vastus lateralis were significantly inverse correlated with $\mathrm{DE}$ unlike to others parameters of whole body cycling efficiency (GE and NE) during an incremental graded exercise test. Therefore, cyclists who showed the highest DE with the largest decrease of skin temperature over quadriceps muscle during the GXT are probably characterised by a better thermoregulation function during exercise. Further research is required to correlate skin infrared thermography and core body temperature which is important in the study of human temperature regulation in daily life. Taking rectal temperature is binding during the cycling exercise. We will use ingestible capsule temperature system $[38,39]$. But we have ensured that the subjects did not consume drinks during the test in order to avoid measurement errors. Measurement of muscle temperature could also be very interesting to effectively ascertain that measures from skin temperature were associated to the thermoregulation process but remain difficult to perform it due to its invasive nature.

\section{Acknowledgements}

The authors wish to thank all the cyclist who have participated to this study and Mr Tschens, headmaster of the cycling center of high school Arago in Reims (France).

\section{References}

1. Gaesser GA, Brooks GA. Muscular efficiency during steady-rate exercise: effects of speed and work rate. J Appl Physiol. 1975; 38(6):1132-9.

2. Ettema G, Lorås HW. Efficiency of cycling: a review. Eur J Appl Physiol. 2009; 106(1):1-14. doi: 10.1007/s00421-009-1008-7.

3. Moseley L, Jeukendrup A. The reliability of cycling efficiency. Med Sci Sports Exerc. 2001; 33(4):621-7.

4. Coyle EF, Sidossis LS, Beltz JD. Cycling efficiency is related to the percentage of type I muscle fibers. Med Sci Sports Exerc. 1992; 24(7):782-8.

5. Brengelmann GL, Johnson JM, Hermansen L, Rowell LB. Altered control of skin blood flow during exercise at high internal temperatures. J Appl Physiol Respir Environ Exerc Physiol. 1977; 43(5):790-4.

6. Hildebrandt C, Zeilberger K, Ring EFJ, Raschner C. The application of medical infrared thermography in sports medicine. In: Zaslav KR, editor. An International Perspective on Topics in Sports Medicine and Sports Injury. Europe: INTECH Open Access Publisher; 2012. p. 25774. DOI: $10.5772 / 28383$.

7. Merla A, Mattei PA, Di Donato L, Romani GL. Thermal imaging of cutaneous temperature modifications in runners during graded exercise. Ann Biomed Eng. 2010; 38(1):158-63. doi: 10.1007/s10439009-9809-8.

8. Nakayama T, Ohnuki Y, Niwa K. Fall in skin temperature during exercise. Jpn J Physiol 1997; 27(4):423-37.

9. Nakayama T, Ohnuki Y, Kanosue K. Fall in skin temperature during exercise observed by thermography. Jpn J Physiol. 1981; 31(5):75762.
10. Abate M, Di Carlo L, Di Donato L, Romani GL, Merla A. Comparison of cutaneous termic response to a standardized warm up in trained and untrained individuals. J Sports Med Phys Fitness. 2013; 53(2):209-15.

11. Arfaoui A, Bertucci W, Polodori G. Thermoregulation during incremental exercise in masters cycling. J Sci Cycling. 2014; 3(1):3341.

12. Clark RP, Mullan BJ, Pugh LG. Skin temperature during running-a study using infra-red colour thermography. J Physiol. 1977; 267(1):53-62.

13. Torii M, Yamasaki M, Sasaki T, Nakayama H. Fall in skin temperature of exercising man. Br J Sports Med. 1992; 26(1):29-32.

14.Vainer BG. FPA-based infrared thermography as applied to the study of cutaneous perspiration and stimulated vascular response in humans. Phys Med Biol. 2005; 50(23):R63-94.

15. James CA, Richardson AJ, Watt PW, Maxwell NS. Reliability and validity of skin temperature measurement by telemetry thermistors and a thermal camera during exercise in the heat. J Therm Biol. 2014; 45:141-9. doi: 10.1016/j.jtherbio.2014.08.010.

16. Chudecka M, Lubkowsak A. The use of thermal imaging to evaluate body temperature changes of athletes during training and a study on the impact of physiological and morphological factors on skin temperature. Human Movement. 2012; 13(1):33-9. DOI: 10.2478/ v10038-012-0002-9.

17. Quesada PJI, Carpes FP, Bini RR, Palmer RS, Pérez-Soriano P, Cibrián Ortiz de Anda RM. Relationship between skin temperature and muscle activation during incremental cycle exercise. J Therm Biol. 2015; 48:28-35. doi:10.1016/j.jtherbio.2014.12.005.

18. Kenney WL, Johnson JM. Control of skin blood flow during exercise. Med Sci Sports Exerc. 1992; 24(3):303-12.

19. Johnson JM. Exercise and the cutaneous circulation. Exerc Sport Sci Rev. 1992; 20:59-97.

20. Johnson JM, Rowell LB, Brengelmann GL. Modification of the skin blood flow-body temperature relationship by upright exercise. J Appl Physiol. 1974; 37(6):880-6.

21. Merla A, Iodice P, Tangherlini A, De Michele G, Di Romualdo S, Saggini $\mathrm{R}$, et al. Monitoring skin temperature in trained and untrained subjects throughout thermal video. Conf Proc IEEE Eng Med Biol Soc. 2005; 2:1684-6.

22. Pellaton C, Kubli, S, Feihl F, Waeber B. Blunted vasodilatory responses in the cutaneous microcirculation of cigarette smokers. Am Heart J. 2002; 144(2):269-74

23. Ihsan M, Landers G, Brearley M, Peeling P. (2010). Beneficial effects of ice ingestion as a precooling strategy on $40-\mathrm{km}$ cycling time-trial performance. Int J Sports Physiol Perform. 2010; 5(2):140-51.

24. Siegel R, Laursen PB. Keeping your cool: possible mechanisms for enhanced exercise performance in the heat with internal cooling methods. Sports Med. 2012; 42(2):89-98. doi: 10.2165/11596870000000000-00000.

25. Naito K, Komori M, Kondo Y, Takeuchi M, Iwata S. The effect of menthol stimulation of the major palatine nerve on subjective and objective nasal patency. Auris Nasus Larynx. 1997; 24(2):159-62.

26. Eccles R. Role of cold receptors and menthol in thirst, the drive to breathe and arousal. Appetite. 2000; 34(1):29-35.

27. Harriss DJ, Atkinson G. (2014). Ethical Standards in Sport and Exercise Science Research: 2014 Update. Int J Sports Med 2014; 34(12):10258. doi: $10.1055 / \mathrm{s}-0033-1358756$. 
28. Hopker J, Passfield L, Coleman D, Jobson S, Edwards L, Carter H. The effect of training on gross efficiency in cycling: a review. Int J Sports Med. 2009; 30(12):845-50. doi: 10.1055/s-0029-1237712.

29. Bertucci W. Analysis of the agreement between the Fortius cycling ergometer and the Power Tap powermeter PO during time trials of 6 and 30 min. Comput Methods Biomech Biomed Engin. 2012; 15(suppl 1):212-4.

30. Peiffer JJ, Losco B. Reliability/Validity of the fortius trainer. Int J Sports Med. 2011; 32(5):353-6. doi: 10.1055/s-0031-1271772.

31. Brouwer E. On simple formulae for calculating the heat expenditure and the quantities of carbohydrate and fat oxidized in metabolism of men and animals, from gaseous exchange (oxygen and carbonic acid output) and urine-N. Acta Physiol Pharmacol Neerl. 1957; 6:795-802.

32. Chavarren J, Calbet JA. Cycling efficiency and pedalling frequency in road cyclists. Eur J Appl Physiol Occup Physiol. 1999; 80(6):555-63.

33. Marsh AP, Martin PE, Foley KO. Effect of cadence, cycling experience, and aerobic power on delta efficiency during cycling. Med Sci Sports Exerc. 2000; 32(9):1630-4.
34. Samozino P, Horvais N, Hintzy F. Why does power output decrease at high pedaling rates during sprint cycling? Med Sci Sports Exerc. 2007; 39(4):680-7.

35. Sidossis LS, Horowitz JF, Coyle EF. Load and velocity of contraction influence gross and delta mechanical efficiency. Int J Sports Med. 1992; 13(5):407-11.

36. Nickleberry BL Jr, Brooks GA. No effect of cycling experience on leg cycle ergometer efficiency. Med Sci Sports \& Exerc. 1996; 28(11):1396-401.

37. De Koning JJ, Noordhof DA, Lucia A, Foster C. (2012). Factors affecting gross efficiency in cycling. Int J Sports Med. 2012; 33(11):880-5. doi: 10.1055/s-0032-1306285.

38. Easton C, Fudge BW, Pitsiladis YP. Rectal, telemetry pill and tympanic membrane thermometry during exercise heat stress. J Therm Biol. 2007; 32(2):78-86. doi:10.1016/j.jtherbio.2006.10.004.

39. Engels HJ, Yarandi HN, Davis JE. Utility of ingestible capsule for core temperature measurements during body warming. J Exercise Physiol online. 2009. 12(1):1. 